\title{
A Dual Compression Ethernet Camera Solution for Airborne Applications
}

\author{
Stephen Willis ${ }^{1}$ and Bernd Langer ${ }^{2}$ \\ ${ }^{1}$ Product Marketing Specialist, Curtiss-Wright Defense Solutions, Dublin, Ireland, \\ swillis@curtisswright.com \\ ${ }^{2}$ Kappa Optronics GmbH, Gleichen, Germany
}

\begin{abstract}
Camera technology is now ubiquitous with smartphones, laptops, automotive and industrial applications frequently utilizing high resolution imagine sensors. Increasingly there is a demand for high-definition cameras in the aerospace market - however, such cameras must have several considerations that do not apply to average consumer use including high reliability and being ruggedized for harsh environments.
\end{abstract}

A significant issue is managing the large volumes of data that one or more HD cameras produce. One method of addressing this issue is to use compression algorithms that reduce video bandwidth. This can be achieved with dedicated compression units or modules within data acquisition systems. For flight test applications it is important that data from cameras is available for telemetry and coherently synchronized while also being available for storage. Ideally the data in the telemetry steam should be highly compressed to preserve downlink bandwidth while the recorded data is lightly compressed to provide maximum quality for onboard/ post flight analysis.

This paper discusses the requirements for airborne applications and presents an innovative solution using Ethernet cameras with integrated compression that outputs two steams of data. This removes the need for dedicated video and compression units while offering all the features of such including switching camera sources and optimized video streams.

Key words: Ethernet, video, camera, compression, flight test

\section{Introduction}

Digital cameras have become ubiquitous in recent years as their prices have dropped. Today, almost every smartphone and laptop, as well as an increasing amount of cars and even children's toys, have a camera sensor. Vendors like GoPro have also developed more rugged cameras intended for outdoor activities while providing high-definition images. High-definition cameras for flight test have yet to become so widespread despite the desirability of having several cameras monitoring surfaces, carriage and internal systems. One of the key reasons for this is that while commercially available camera technology can be used in some limited capacity, they are not specifically designed for flight test applications.

Broadly speaking, the requirements for flight test cameras can be split into two categories camera characteristics and system level design and integration.

\section{Camera characteristics}

Camera characteristics describe the physical elements a flight testing camera should have in order to meet both image and environmental requirements. Just as other sensors, data acquisition units or recording equipment are built according to specific flight testing requirements, cameras should also be designed with the application in mind. The most important of these considerations include the following aspects.

\section{Signal quality requirements}

Signal quality starts at image capture. Not all digital sensors / processing hardware can meet required image capture. For example, for flight tests it's important to have a 'global shutter' to eliminate the smearing effect caused by a 'rolling shutter' in Figure 1. 

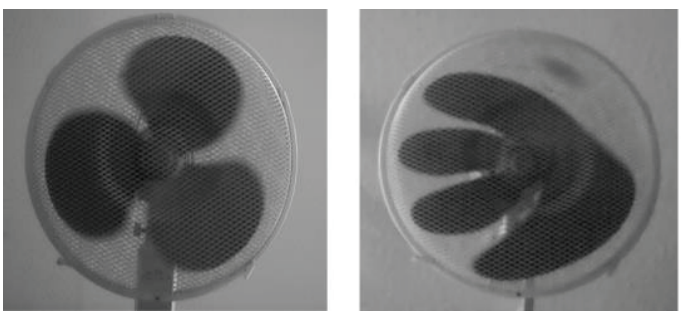

Figure 1: Global shutter (clear) and rolling shutter (distorted)

The resolution of the CCD sensor and the color processing of a camera, are also important aspects for a proper image quality.

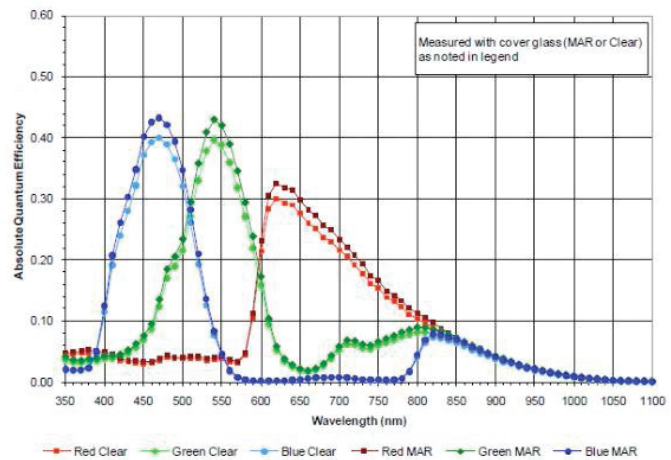

Figure 2: Spectral sensitivity of a typical $C C D$

HD-resolution (High-definition) helps to increase the viewing angle without losing image quality. The human eye has a field of view of approx. $50^{\circ}$ at an angular resolution of $\sim 1^{\prime}$.
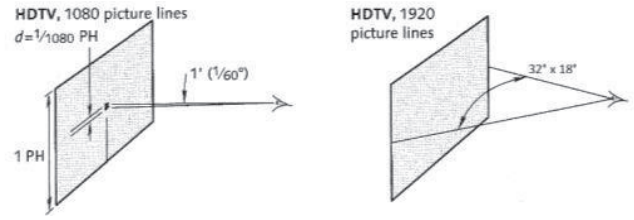

Figure 3: Viewing angles at $H D$ resolution

A standard full HD sensor with a resolution of $1920 \times 1080$ pixels at an angular resolution of $1^{\prime}$ results in a horizontal viewing angle of $32^{\circ}$; while an SD-sensor (Standard Definition) with $720 \times 576$ pixels results in a viewing angle of only $12^{\circ}$.

Other important aspects during image capture include low noise readout, black level control, adjustable exposure time, gain, color settings amongst others. Therefore the following imageprocessing functions are essential for appropriate image quality during test flights:

- Defect pixel correction

- Automatic exposure control

- Automatic gain control

- Automatic white balance control

- Lens distortion correction

\section{Meeting external environmental conditions}

Factors such as vibration, shock, humidity and temperature could damage or reduce the effectiveness of the units. Therefore cameras have to be designed to meet the strong environmental requirements.

Table 1: External Environmental Requirements

\begin{tabular}{|c|c|}
\hline Temperature range & $-55^{\circ} \mathrm{C}$ to $+85^{\circ} \mathrm{C}$ \\
\hline Altitude & $50.000 \mathrm{ft}(116 \mathrm{mbar}$ ) \\
\hline Humidity & $\begin{array}{c}\text { Cycles between } 95 \% / \\
65^{\circ} \mathrm{C} \text { and } 85 \% / 38^{\circ} \mathrm{C} \\
\text { (for } 16 \mathrm{hours} \text { ) }\end{array}$ \\
\hline EMI (emissions) & $10 \mathrm{kHz}$ to $6 \mathrm{GHz}$ \\
\hline EMI (susceptibility) & $100 \mathrm{MHz}$ to $6 \mathrm{GHz}$ \\
\hline $\begin{array}{c}\text { Lightning, indirect } \\
\text { effect }\end{array}$ & up to $1600 \mathrm{~V} / 107 \mathrm{~A}$ \\
\hline Shock & $40 \mathrm{~g} / 10 \mathrm{~ms}$ \\
\hline Vibration & $6 \mathrm{grms}, 5-2000 \mathrm{~Hz}$ \\
\hline
\end{tabular}

Rugged optical entrance windows are required to protect against scratches and breakage sapphire glass has these properties. A method to prevent icing and misting on the lens, such as a heating element is also recommended.

For safe handling of a camera in the flight test environment, ruggedized connectors help to maximize the availability of the camera.

To ensure that these requirements are met, the camera has to be qualified (DO-160, MILSTD461, MIL-STD 464, MIL-STD 810, MIL-STD 704) at certified laboratories.

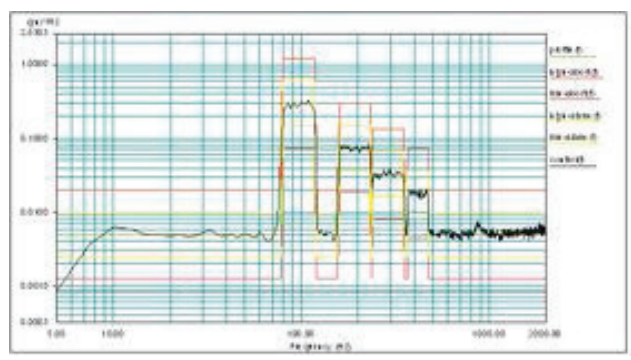

Figure 4: Random Vibration Test

But also at quality management (ISO 9100, EN/AS 9100) and Manufacturing (IPC-A-610 E, IPC-A-600, IPC J-STD-011, IPC/WHMAA-A620) a lot of standards have to be taken into account.

\section{Matching to flight test mission}

Each flight test mission has its own needs. For example a wide range of lenses are needed to get the important information at maximum 
resolution. Viewing angles from $10^{\circ}$ for small objects far away, up to $95^{\circ}$ to get a wide scene overview are useful. An appropriate lens should have adjustable focus plane and aperture.

Also an easy mounting mechanical interface is important. The camera should be easily mounted in a secure manner with the ability to fine tune the direction of observation.

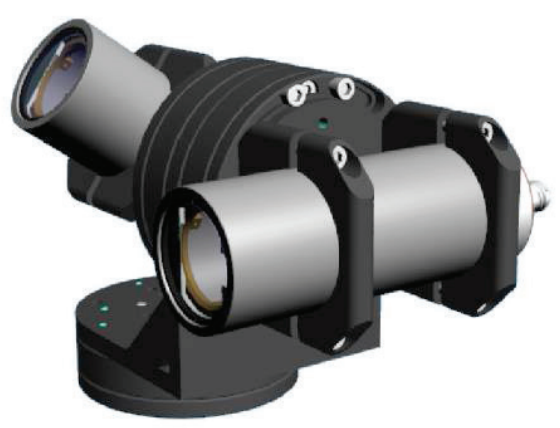

Figure 5: Mounting Kit

All adjustable camera features have to be configurable in a way that doesn't require further actions during a test flight.

\section{System Level Characteristics}

At a system level, data from the camera is typically sent to ground via a telemetry bridge and to a recording device. This is relatively straight forward for a single high-definition or a few standard definition cameras. The onboard data acquisition system is likely able to support such via video compression cards. However, once several high-definition cameras are required the bandwidth required for uncompressed video can overload the data acquisition system e.g. full HD video at $60 \mathrm{fps}$ can take up to $3 \mathrm{Gbps}$ of bandwidth per channel as shown in Table 2.

Table 2: Bandwidth requirements for uncompressed video

\begin{tabular}{|c|c|c|c|c|}
\hline Format & $\begin{array}{c}\text { Pixels } \\
{[\mathrm{M}]}\end{array}$ & $\begin{array}{c}\text { Frame } \\
\text { rate } \\
\text { [fps] }\end{array}$ & $\begin{array}{c}\text { Color } \\
\text { depth } \\
\text { [bit] }\end{array}$ & $\begin{array}{c}\text { Bandwidth } \\
\text { [Gbps] }\end{array}$ \\
\hline SD 576 & .414 & 24 & 24 & 0.24 \\
\hline HD 720p & .922 & 30 & 24 & 0.66 \\
\hline HD 1080p & 2.07 & 30 & 24 & 1.5 \\
\hline HD 1080p & 2.07 & 60 & 24 & 3 \\
\hline
\end{tabular}

One method of solving this problem is to use dedicated video multiplexors and management units to isolate the camera network from the FTI system. One can then use a dedicated video recorder to store uncompressed video data and selectively send channels of interest to ground via a telemetry bridge. Figure 6 shows an example of such a system. However, often these video management units limit the data from cameras as the telemetry system and the recorder required to handle such raw data rates can be expensive, large and heavy. A work around is to lower the frame rate, e.g. send only every tenth frame, or the color depth e.g. a monochrome image might yield the data required.

In addition, setting up and managing the video management unit can be difficult as they can be complex when trying to direct limited data to different locations. Another issue is that the video is not synchronized with other FTI parameters resulting in time consuming post processing work if correlation between the video and other data is required. The use of separate boxes also adds weight and reduces available space.

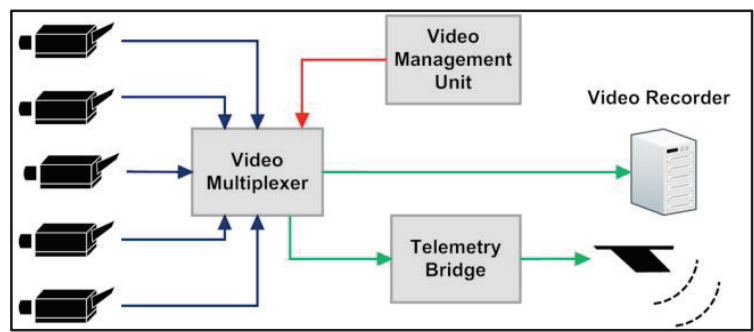

Figure 6: Camera system using video multiplexors and management unit

A method to overcome some of these problems is to use video compression. As raw HD video can be over 1 Gbps, more bandwidth can be taken by one video channel than the entire data acquisition of a very large network. Compressing this data using an industry standard algorithm can reduce the bandwidth to a more reasonable amount without significantly affecting the image quality.

Often video data contains a lot of redundancy such as intra and inter frame redundancy. Intra frame redundancy is where one part of frame is similar to another or intra frame redundancy where successive frames are similar to each other. Figure 7 shows an example of an aircraft wing where there are areas which are very similar to each other, such as sections of sky and the wing (intra frame redundancy), and sections which will change little between frames (inter frame redundancy). 


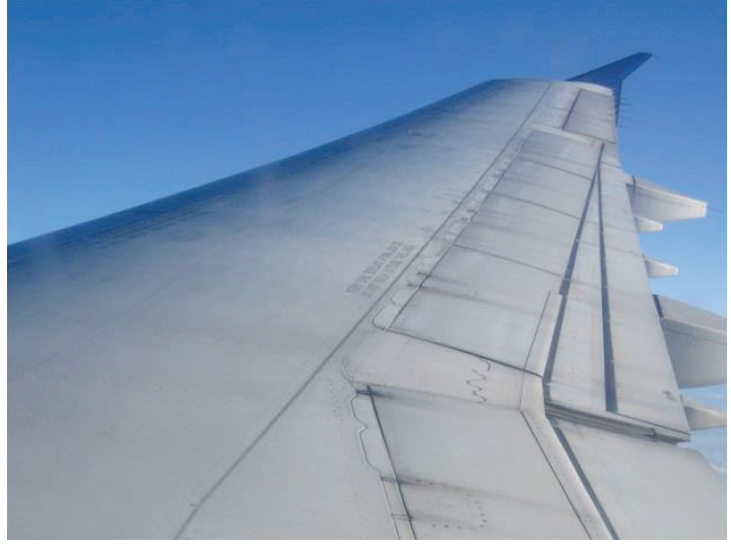

Figure 7: Video data can contain significant redundancy

A compression algorithm such as MPEG 4 or H.264 can exploit both these redundancies whereas compression algorithms such as JPEG 2000 or MPEG 2 rely exclusively on intra frame redundancy. The advantage of only using intra frame redundancy is it reduces latency in the compression hardware and it is more robust against corruption due to signal drop outs (if an intra coded frame ('l' frame) is lost then the predicted frames ('P' frames) will be corrupted).

In practice these effects can be minimized with modern hardware and by selecting appropriate trade-offs between high compression and robustness against signal drop-out. Figure 8 shows how such an inter frame scheme can be setup where the $P$ frames reference the previous frame to remove redundancy. In a scheme such as H.264, it is possible to only have I frames which effectively makes the codec inter only and perform very much like JPEG 2000.

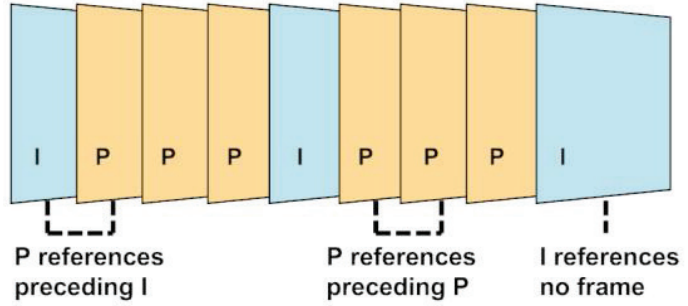

Figure 8: I and $P$ frames in a compression algorithm

Using a modern video compression/ decompression algorithm (codec) to exploit such redundancy can result in a HD video stream that can be compressed down to $<10$ Mbps. Thus bandwidth and recording capacity required to handle compressed video can be over 100 times less than for raw video, often without a great loss in quality. This compression can be performed by a video multiplexing unit, however the unit will need to be powerful as it must be capable of handling multiple high bandwidth inputs and perform compression on these. Thus the unit is likely to be expensive, large and heavy.

An alternate solution is to perform the compression at source i.e. in the cameras. This has the advantage of splitting the compression task into less complex sub-units that inherently scale with the number of cameras used. This also means that there is no longer any need for a dedicated video multiplexor or management unit. Using Ethernet as the data transfer standard, a COTS switch can be used to route the packetized video data to the destination devices. Ethernet has the additional benefit of allowing packets to be filtered so a standard PC can be used to 'switch' camera source onboard as well as monitoring other FTI parameters.

A key benefit of such a camera design is that the onboard compression and packet based transmission facilitates multiple compression streams. This is particularly of use for flight test instrumentation where being able to define two compression rates for the same channel over the same Ethernet connection allows the user to set one data rate for the recorder and a second data rate for PCM transmission (Figure 9). Filtering in the switch, either by port or by packet header, routes lightly compressed data to a recorder while highly compressed data is sent into the FTI system. Time stamping, such as the IEEE-1588 Precision Time Protocol (PTP), can be used to synchronize the video packets with other parameters in the FTI system.

As a result, the video recorded is of good quality and high resolution whereas the video sent over PCM is of a lower quality, but allows the user to save on PCM bandwidth and send multiple video signals on a single telemetry link.

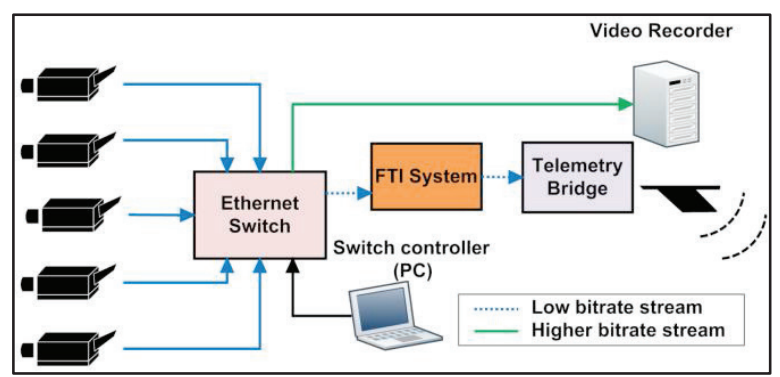

Figure 9: Dual compression on FTI cameras 


\section{IRIS Airborne Camera}

The IRIS airborne camera (Figure 10) from Curtiss-Wright was developed to address the future high-definition video needs for flight test applications. It is a rugged FTI camera which uses H.264 compression to deliver two channels of video over a single Ethernet link. It can be seamlessly integrated with the Acra KAM-500 data acquisition product line or used in a stand-alone system. The camera can be connected directly to an Ethernet switch, removing the requirement for a dedicated compression module. As there is no need for dedicated hardware compression, SWaP (Size Weight and Power) is minimized.

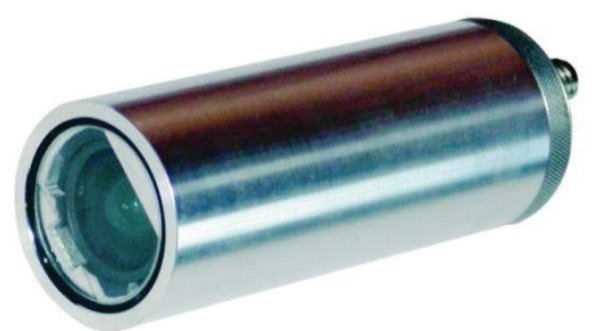

Figure 10: The IRIS airborne camera

The camera has two compression streams; a primary stream for recording, and a secondary preview stream for telemetry. The preview stream is more highly compressed, reducing the telemetry bandwidth required for transmission of data to the ground. As with Ethernet switches and network recorders, the camera can be set up and configured using the same data acquisition software reducing time and effort in configuring the system. Video data captured can be replayed using commercial offthe-shelf software or flight test replay and analysis software.

The camera's data can be timestamped using a PTPv1 or a PTPv2 Grandmaster. This allows the acquired video to be timestamped using the same mechanism as all other parameters in the FTI network. Other useful features for flight test applications include

- Different Lenses of focal length between 4.8 $\mathrm{mm}$ and $35 \mathrm{~mm}$

- Full HD 1080P color (1 to $30 \mathrm{fps}$ )

- RTSP, RTP and iNET-X compliant dual video streaming

- Unicast as well as Multicast Streaming

- High quality Kodak CCD sensor with global shutter imager

- Rugged housing designed for the environmental conditions of flight test

- Playback with off the shelf players or with flight test software for synchronized video and other FTI parameters

\section{Conclusions}

Flight test applications benefit from cameras designed specifically to meet the application and environmental requirements. Such cameras perform more reliably and integrate with other FTI better than consumer products which were not designed for the task.

Video cameras are moving from $S D$ to $H D$ which presents issues for existing video system solutions. Transmitting raw video adds complexity and limits the amount of video frames that can be transmitted and stored. Synchronizing this video data with other flight test parameters can also be a challenge. Dedicated compression cards can solve some of these issues but they have implications for SWaP.

Ethernet cameras with integrated compression aim to address these issues with dual output streams to suit recorder and telemetry applications, capabilities to switch video streams in an Ethernet switch and synchronization between FTI and video data. The use of simple and consistent setup software and off-the-shelf technologies facilitate quick and easy deployment. 